\title{
THE LEFSCHETZ NUMBER OF SELF-MAPS OF LIE GROUPS
}

\author{
DUAN HAIBAO
}

(Communicated by Haynes R. Miller)

\begin{abstract}
In this note we present a simple approach to the Lefschetz number for the self-maps of Lie groups. As an application it is proved that for any map $f: G \rightarrow G$ of a compact connected Lie group $G$, there is a solution to $(f(x))^{k}=x$ for some $k \leq \lambda+1$, where $\lambda$ is the rank of the group $G$.
\end{abstract}

Let $G$ be an $n$-dimensional compact connected Lie group with multiplication $\mu$, inverse $T$ and unit $e$. Let $[G, G]$ be the set of homotopy classes of maps $G \rightarrow G$. Given two maps $f, f^{\prime}: G \rightarrow G$, we write $f \cdot f^{\prime}$ to denote the map $G \rightarrow G$ defined by

$$
\left(f \cdot f^{\prime}\right)(g)=\mu\left(f(g), f^{\prime}(g)\right), \quad g \in G .
$$

All cohomology in this paper will be over a coefficient field $F$ of zero characteristic.

Let Lef: $[G, G] \rightarrow Z$ be the function that sends each element in $[G, G]$ to its Lefschetz number. Then the classical Lefschetz fixed point theorem states that "if $f: G \rightarrow G$ is a map with $\operatorname{Lef}(f) \neq 0$, then $f$ has a fixed point." Now we define another function $B:[G, G] \rightarrow Z$ by setting $B(f)=$ degree $f \cdot T$. Since $B(f) \neq 0$ implies $e \in \operatorname{Im} f \cdot T$, this function also possesses the property that "if $B(f) \neq 0, f$ has a fixed point." The following assertion was suggested by Professor Jiang Boju.

THEOREM 1. The two functions Lef, $(-1)^{n} B:[G, G] \rightarrow Z$ coincide.

Before setting out to prove this result, we fix some notation and recall some facts about Lie groups. Given a point $g \in G$ and a differentiable map $F: G \rightarrow G$, write $G_{g}$ to denote the tangent space to $G$ at $g$ and $d_{g} F$, the differential of $F$ at $g$. Let $L_{g}, R_{g}: G \rightarrow G$ be respectively the left translation $L_{g}\left(g^{\prime}\right)=\mu\left(g, g^{\prime}\right)$, and the right translation $R_{g}\left(g^{\prime}\right)=\mu\left(g^{\prime}, g\right)$. Then there is a natural homomorphism Ad, the adjoint representation, from $G$ to $\operatorname{GL}\left(G_{e}\right)$ (the nonsingular linear transformation group of $G_{e}$ ) defined as follows:

$$
\operatorname{Ad}(g)=d_{g} R_{g^{-1}} \circ d_{e} L_{g}=d_{g^{-1}} L_{g} \circ d_{e} R_{g^{-1}} .
$$

Since $G$ is connected the image of Ad belongs to the connected component of $\operatorname{GL}\left(G_{e}\right)$ containing the identity, i.e. for each $g \in G$, $\operatorname{det} \operatorname{Ad}(g)>0$. By Exercise $\mathrm{A} 1$ in

Received by the editors October 14, 1987.

1980 Mathematics Subject Classification (1985 Revision). Primary 55M20.

Key words and phrases. Lefschetz number, mapping degree, fixed point, Lie group, adjoint representation, exterior algebra, primitive elements in the cohomology of a Lie group, Vandermonde matrix.

This work is partially supported by a TWAS grant. 
$[1$, p. 147] we have

LEMMA 1. $d_{g} T=-d_{e} L_{g^{-1}} \circ d_{g} R_{g^{-1}}=-d_{e} R_{g^{-1}} \circ d_{g} L_{g^{-1}} ;$

$$
d_{\left(g_{1}, g_{2}\right)} \mu(X, Y)=d_{g_{2}} L_{g_{1}}(Y)+d_{g_{1}} R_{g_{2}}(X), \quad(X, Y) \in G_{g_{1}} \times G_{g_{2}} .
$$

ProOF OF THEOREM 1. For each $g \in G$, we identify $G_{g}$ with $G_{e}$ by the differential of the left translation $L_{g}$ at $e$. Let $I_{g}: G_{g} \rightarrow G_{g}$ be the identity map.

For any element in $[G, G]$, we can choose a representative $f: G \rightarrow G$ such that

(1) $f$ is differentiable;

(2) $f$ has only finitely many fixed points $g_{1}, \ldots, g_{k}$;

(3) $\operatorname{det}\left(I_{g_{i}}-d_{g_{i}} f\right) \neq 0$.

Then a discussion in [2, p. 139] shows that

$$
\operatorname{Lef}(f)=\sum_{1}^{k} \operatorname{sign} \operatorname{det}\left(I_{g_{i}}-d_{g_{i}} f\right) .
$$

$(f \cdot T)^{-1}(e)=\left\{g_{1}, \ldots, g_{k}\right\}$, and for each $i$ the differential of $(f \cdot T) \circ L_{g_{i}}$ at $e$ is the composite

$$
G_{e} \stackrel{d L_{g}}{\longrightarrow} G_{g_{i}} \stackrel{d \Delta}{\longrightarrow} G_{g_{i}} \times G_{g_{i}} \stackrel{d f \times d T}{\longrightarrow} G_{g_{i}} \times G_{g_{i}^{-1}} \stackrel{d \mu}{\longrightarrow} G_{e},
$$

where $\Delta: G \rightarrow G \times G$ is the diagonal map. It follows from Lemma 1 that the above homomorphism is just the same as

$$
\operatorname{Ad}\left(g^{-1}\right)\left(d_{e}\left(L_{g_{i}^{-1}} \circ f L_{g_{i}}\right)-I_{e}\right): G_{e} \rightarrow G_{e} .
$$

Denote the map by $A_{i}$. Then by the convention (3) $\operatorname{det} A_{i} \neq 0$ and

$$
(-1)^{n} \operatorname{sign} \operatorname{det} A_{i}=\operatorname{sign} \operatorname{det}\left(I_{g_{i}}-d_{g_{i}} f\right) \text {. }
$$

So we see that $e$ is a regular value of $f \cdot T$ and

$$
B(f)=\sum_{1}^{k} \operatorname{sign} \operatorname{det} A_{i}=(-1)^{n} \operatorname{Lef}(f) .
$$

This completes the proof.

To show the implications of the above theorem, recall that $H^{*}(G ; F)$ is an exterior algebra $\Lambda\left(x_{1}, \ldots, x_{\lambda}\right)$ generated by primitive elements $x_{i}$ of odd degree [3] with $\lambda=\operatorname{rank} G$. Also from [3] we have

LEMMA 2. If $f, f^{\prime}: G \rightarrow G$ are two maps, and if $x \in H^{*}(G ; F)$ is primitive, then $\left(f \cdot f^{\prime}\right)^{*}(x)=f^{*}(x)+f^{\prime *}(x)$.

REMARK. It immediately follows from Lemma 2 that $\operatorname{Lef}\left(f \cdot f^{\prime}\right)=\operatorname{Lef}\left(f^{\prime} \cdot f\right)$.

Since Id $\cdot T: G \rightarrow G$ is the map collapsing $G$ to unit $e$, by the lemma above $T^{*}\left(x_{i}\right)=-x_{i}$. Let $\cup$ be the cup product in $H^{*}(G ; F)$. Then Lemma 2 also implies

$$
\begin{aligned}
B(f)^{*}\left(x_{1} \cup \cdots \cup x_{\lambda}\right) & =(f \cdot T)^{*}\left(x_{1} \cup \cdots \cup x_{\lambda}\right) \\
& =\left(f^{*}\left(x_{1}\right)-x_{1}\right) \cup \cdots \cup\left(f^{*}\left(x_{\lambda}\right)-x_{\lambda}\right) .
\end{aligned}
$$

Since $n=\lambda \bmod 2$, we can rewrite Theorem 1 as follows:

THEOREM 2. $\operatorname{Lef}(f) x_{1} \cup \cdots \cup x_{\lambda}=\left(x_{1}-f^{*}\left(x_{1}\right)\right) \cup \cdots \cup\left(x_{\lambda}-f^{*}\left(x_{\lambda}\right)\right)$.

Given a map $f: G \rightarrow G$ and an integer $k>0$, let ${ }^{k} f$ be the $k$-fold product of $f$ defined inductively by ${ }^{1} f=f,{ }^{k} f=f \cdot{ }^{k-1} f$. 
COROLlaRY 1. For any map $f: G \rightarrow G$, there is an integer $k$ with $0<k \leq \lambda+1$ such that $\operatorname{Lef}\left({ }^{k} f\right) \neq 0$.

ProOF. Given a map $h: G \rightarrow G$, regard the expression

$$
\left(x_{1}-h^{*}\left(x_{1}\right)\right) \cup \cdots \cup\left(x_{\lambda}-h^{*}\left(x_{\lambda}\right)\right)
$$

as a formal polynomial in the elements $x_{i}$ and $h^{*}\left(x_{j}\right)$. For each integer $t$ with $0 \leq t \leq \lambda$, there exists an element $A_{t}(h)$ in $F$ such that $A_{t}(h) x_{1} \cup \cdots \cup x_{\lambda}=$ sum of the monomials appearing in above polynomial, and containing just $t$ elements in $h^{*}\left(x_{j}\right)$. Then Lemma 2 and Theorem 2 imply that

$$
\operatorname{Lef}\left({ }^{k} f\right)=\sum_{0}^{\lambda} k^{t} A_{t}(f) \text { for any } k>0 .
$$

So if $H=\left(a_{s t}\right)$ is the $(\lambda+1) \times(\lambda+1)$ Vandermonde matrix defined by $a_{s t}=t^{s-1}$, $1 \leq s, t \leq \lambda+1$, then

$$
\left(\operatorname{Lef}(f), \operatorname{Lef}\left({ }^{2} f\right), \ldots, \operatorname{Lef}\left({ }^{\lambda+1} f\right)\right)=\left(A_{0}(f), A_{1}(f), \ldots, A_{\lambda}(f)\right) H .
$$

Now Corollary 1 follows from $\operatorname{det} H \neq 0$ and $A_{0}(f)=1 \neq 0$.

Suppose $h: G \rightarrow G$ is a homomorphism. Then for any primitive element $x \in$ $H^{*}(G ; F), h^{*}(x)$ is also primitive. Since the primitive elements form a submodule of $H^{*}(G ; F)$ with basis $\left\{x_{1}, \ldots, x_{\lambda}\right\}$, there exists a $\lambda \times \lambda$ matrix $M_{h}$ over $F$ such that

$$
\left(h^{*}\left(x_{1}\right), \ldots, h^{*}\left(x_{\lambda}\right)\right)=\left(x_{1}, \ldots, x_{\lambda}\right) M_{h}
$$

By Theorem 2 a discussion on linear algebra can show

COROLlaRY 2. Lef $(h)=\operatorname{det}\left(I-M_{h}\right)$.

Theorem 2 also leads to an easier way to make the following well-known computation:

COROLlaRY 3. $\operatorname{Lef}\left({ }^{k} \mathrm{Id}\right)=(1-k)^{\lambda} ; \operatorname{Lef}\left({ }^{k} T\right)=(1+k)^{\lambda}$.

The significance of it has been discussed in [4].

COMMENT. In fact Theorem 2 also has a purely cohomological proof without using Theorem 1. So all results, except Theorem 1, are valid for self-maps of a compact connected triangulable $H$-group.

ACKNOWLEDGMEnT. I am very grateful to Professor Jiang Boju who has given me help, suggestions, and criticism while writing this note.

\section{REFERENCES}

1. S. Helgason, Differential geometry, Lie group and symmetric spaces, Academic Press, New York, 1978.

2. M. W. Hirsch, Differential topology, Springer-Verlag, Berlin and New York, 1978.

3. G. W. Whitehead, Elements of homotopy theory, Springer-Verlag, Berlin and New York, 1978.

4. R. F. Brown, The Lefschetz fixed point theorem, Scott-Foresman, 1971.

Department of Mathematics, Peking University, Peking, China

Current address: Institute of Systems Science, Academia Sinica, Beijing 100080, China 